\title{
Processed meat consumption and risk of cancer: a multisite case-control study in Uruguay
}

\author{
E De Stefani*,', P Boffetta ${ }^{2}$, AL Ronco ${ }^{3}$, H Deneo-Pellegrini', P Correa ${ }^{4}$, G Acosta', M Mendilaharsu', \\ ME Luaces' and C Silva'
}

'Epidemiology Group, Department of Pathology, School of Medicine, University of the Republic, Montevideo, Uruguay; ${ }^{2}$ The Tisch Cancer Institute, Mount Sinai School of Medicine, New York, NY, USA; ${ }^{3}$ Department of Epidemiology, IUACLAEH University of Maldonado, Maldonado, Uruguay; ${ }^{4}$ Division of Gastroenterology, Hepatology, and Nutrition, Vanderbilt University Medical Center, Nashville, TN, USA

BACKGROUND: The role of processed meat in the aetiology of several cancers was explored in detail.

METHODS: In the time period 1996-2004, a multisite case-control study was conducted in Montevideo, Uruguay. The study included 6060 participants ( 3528 cases and 2532 controls) corresponding to cancers of the oral cavity, pharynx, oesophagus, stomach, colon, rectum, larynx, lung, female breast, prostate, urinary bladder, and kidney (renal cell carcinoma only).

RESULTS: The highest odds ratios (ORs) were positively associated with cancers of the colon, rectum, stomach, oesophagus, and lung. With the exception of renal cell carcinoma, the remaining cancer sites were significantly associated with elevated risks for processed meat consumption. Furthermore, mortadella, salami, hot dog, ham, and salted meat were strongly associated with risk of several cancer sites.

CONCLUSION: It could be concluded that processed meat intake could be a powerful multiorgan carcinogen.

British Journal of Cancer (2012) 1 07, 1584-1588. doi:I0.1038/bjc.2012.433 www.bjcancer.com

Published online 25 September 2012

(c) 2012 Cancer Research UK

Keywords: oesophageal cancer; gastric cancer; colorectal cancer; lung cancer; breast cancer; prostate cancer

Uruguay is a developing country characterised by high incidence rates of cancer (ASR 386.0 cases per 100000 males and 303.2 cases per 100000 females; Parkin et al, 2002). The leading cancer site among men is the lung, whereas the main cancer site among women is the breast, following the pattern of the developed countries. The main reasons for these elevated rates are related to the prevalence of tobacco smoking, alcohol drinking, the high consumption of red meat, and the low consumption of vegetables and fruits.

In fact, the Uruguayan population is characterised by a very high consumption of red meat (the highest in the world (Matos and Brandani, 2002)), a high consumption of processed meat, and a low intake of white meat. Several reports have suggested that processed meat is linked to the aetiology of frequent malignancies like gastric cancer and colon cancer (Larsson et al, 2006; Cross et al, 2007). In fact, the consumption of processed meat is much higher in Uruguay compared with consumption in the United States (Cross et al, 2007).

For this reason, we decided to conduct a multisite case-control study in order to explore the role of processed meat in 11 cancer sites in Uruguay.

\section{MATERIALS AND METHODS}

\section{Selection of cases}

In the time period 1996-2004, all newly diagnosed and microscopically confirmed cases of cancers of the oral cavity, pharynx, oesophagus, stomach, colon, rectum, larynx, lung, female breast,

*Correspondence: Dr E De Stefani; E-mail: edestefani@gmail.com Received 27 June 2012; revised 15 August 2012; accepted 20 August 2012; published online 25 September 2012 prostate, bladder, and kidney were considered eligible for this study (3641 cases). One hundred and thirteen patients refused the interview, leading to a final total of 3528 cases ( 2648 men and 880 women) (response rate: $96.9 \%$ ). All the cases were drawn from the four major public health hospitals of Montevideo. These hospitals admit only patients of low socioeconomic status, with a monthly income $<200$ US dollars.

\section{Selection of controls}

In the same time period and in the same hospitals, all patients with conditions not related to smoking and drinking were considered eligible for the study. In this period, 2608 potential controls were approached for a possible participation and 76 patients refused the interview, leaving a final number of 2532 controls (response rate: 97.1\%). These controls presented the following diseases: eye disorders (622 patients, $24.6 \%$ ), abdominal hernia (513 patients, $20.3 \%$ ), fractures (258 patients, $10.2 \%$ ), injuries (200 patients, $7.9 \%)$, varicose veins (178 patients, $7.0 \%)$, acute appendicitis (176 patients, $6.9 \%)$, diseases of the skin (160 patients, $6.3 \%)$, hydatid cyst (127 patients, 5.0\%), urinary stones (119 patients, $4.7 \%$ ), blood disorders (89 patients, 3.5\%), bone disorders (50 patients, $2.0 \%$ ), and prostate hypertrophy (40 patients, $1.6 \%$ ).

\section{Interviews and questionnaire}

All the participants (6060 cases and controls) were interviewed shortly after admission to the hospital by two trained social workers, unaware of the purposes of the present study. In all the instances, the interview was conducted face to face and proxy interviews were not allowed. The participants were administered a 
structured questionnaire, which included the following sections: (1) a sociodemographic section (age, sex, residence, education, monthly income), (2) an occupational history on the basis of the last four jobs and their duration, (3) self-reported height and weight, 5 years before the date of the interview, (4) a complete smoking history (age at start, age at quit, number of cigarettes smoked per day, type of tobacco, type of cigarette, inhalation practices), (5) a complete alcohol-drinking history (age at start, age at quit, number of glasses drunk per day, type of alcoholic beverage), (6) a complete history of non-alcoholic beverages (age at start, age at quit, number of cups per day, type of beverage: maté, coffee, tea, soft drinks), (7) menstrual and reproductive events, and (8) a food frequency questionnaire (FFQ) on 64 food items. This FFQ was recorded in servings per week and was previously tested for reproducibility with good results (Ronco et al, 2006).

\section{Components of processed meat}

The following meats were analysed in the study: bacon, sausage, mortadella, salami, saucisson, hot dog, ham, and air-dried and salted lamb. These components were energy-adjusted by the residual method (Willett, 1998).

\section{Statistical analysis}

Relative risks, approximated by the OR, were estimated by unconditional logistic multiple regression (Rothman et al, 2008).

Table I Number and percentages by cancer sites and controls

\begin{tabular}{|c|c|c|c|}
\hline Cancer site & $\begin{array}{c}\text { Males } \\
\text { Number (\%) }\end{array}$ & $\begin{array}{c}\text { Females } \\
\text { Number (\%) }\end{array}$ & $\begin{array}{c}\text { Both sexes } \\
\text { Number (\%) }\end{array}$ \\
\hline Oral/pharynx & $274(10.3)$ & $9(1.0)$ & $283(8.0)$ \\
\hline Oesophagus & $184(6.9)$ & $50(5.7)$ & $234(6.6)$ \\
\hline Stomach & $190(7.2)$ & $84(9.5)$ & $274(7.8)$ \\
\hline Colon & $87(3.3)$ & $89(10.5)$ & $176(5.0)$ \\
\hline Rectum & $127(4.8)$ & $58(6.6)$ & 185 (5.2) \\
\hline Larynx & $274(10.3)$ & $7(0.8)$ & 281 (8.0) \\
\hline Lung & $865(32.7)$ & $55(6.3)$ & $920(26.1)$ \\
\hline Breast & - & $46 \mid(52.4)$ & $461(13.1)$ \\
\hline Prostate & $345(13.0)$ & - & $345(9.8)$ \\
\hline Bladder & $225(8.5)$ & $30(3.4)$ & $255(7.2)$ \\
\hline Kidney & $77(2.9)$ & $37(4.2)$ & 144 (3.2) \\
\hline All cases & $2648(100.0)$ & $880(100.0)$ & $3528(100.0)$ \\
\hline All controls & $1640(100.0)$ & $892(100.0)$ & $2532(100.0)$ \\
\hline
\end{tabular}

As the number of controls was smaller than the number of cases, we were unable to use multiple polynomial regression, and the ORs for each cancer site for processed meat intake were estimated by unconditional multiple logistic regression. We fitted the following model for each site including age (continuous), sex (when necessary), residence (urban, rural), education (categorical, 3 strata), body mass index (continuous), smoking index (smoking status, smoking cessation, number of cigarettes smoked per day among current smokers, categorical, 8 strata), alcohol drinking (categorical, 5 strata), maté consumption (categorical, 4 strata), total energy (continuous), total vegetables and fruits (continuous), total white meat (continuous), and red meat (continuous). We then included 3 terms for meat intake (red, processed and white meat) to capture the effect of total meat in each estimation. All the calculations were performed using the STATA software (Stata Corp., College Station, TX, USA), release 11 (StataCorp., 2008).

\section{RESULTS}

The distribution of cases and controls by cancer site, stratified by sex, is shown in Table 1. For both sexes, lung cancer occupied the first place $(26.1 \%)$, followed by breast cancer $(13.1 \%)$, colorectal cancer $(10.2 \%)$, and prostate cancer $(9.8 \%)$.

Cases and controls showed similar age and education, the cases being heavy smokers, and consuming more alcohol and maté than controls (Table 2).

Odds ratios of cancer sites for processed meat intake are shown in Table 3. Cancers of the colon (OR for the highest tertile $v s$ the first tertile 2.75 , 95\% CI 1.80-4.22), rectum (OR 2.14, 95\% CI $1.42-$ 3.22), colorectum (OR 2.39, 95\% CI 1.76-3.24), stomach (OR 2.60, 95\% 1.83-3.70), and larynx (OR 2.37, 95\% CI 1.59-3.53) showed the highest risks. With the exception of oropharyngeal cancer and renal cell carcinoma, the remaining sites displayed a significantly positive association with processed-meat consumption, including cancers of the oesophagus, lung, upper aerodigestive tract, female breast, prostate, and urinary bladder. The model fitted included white meat and red meat intakes, thus it captured total meat in the estimations.

Odds ratios of cancer sites for different types of processed meat are shown in Table 4. Curiously, bacon was inversely associated with most cancer sites and sausage was not associated with the risk of cancer. On the other hand, mortadella intake was positively associated with cancers of the stomach (OR 1.13, 95\% CI 1.021.26 ), colon (OR 1.18, 95\% 1.04-1.34), larynx (OR 1.28, 95\% CI 1.14-1.42), lung (OR 1.15, 95\% CI 1.07-1.23), breast (OR 1.29, 95\% CI 1.16-1.43), and prostate (OR 1.29, 95\% CI 1.16-1.42). Similarly,

Table 2 Means of cancer sites and controls for age, education, smoking intensity, alcohol drinking, and processed meat

\begin{tabular}{|c|c|c|c|c|c|c|c|c|c|c|}
\hline \multirow[b]{2}{*}{ Cancer site } & \multicolumn{2}{|c|}{ Age (years) } & \multicolumn{2}{|c|}{ Education (years) } & \multicolumn{2}{|c|}{$\begin{array}{c}\text { Smoking } \\
\text { (cigarettes per day) }\end{array}$} & \multicolumn{2}{|c|}{$\begin{array}{c}\text { Alcohol } \\
(\mathrm{ml} / \mathrm{ethanol} \text { per day })\end{array}$} & \multicolumn{2}{|c|}{$\begin{array}{l}\text { Processed meat } \\
\text { (grams per day) }\end{array}$} \\
\hline & Men & Women & Men & Women & Men & Women & Men & Women & Men & Women \\
\hline Oral/pharynx & 60.0 & 54.9 & 4.3 & 4.4 & 27.6 & 14.0 & 213.1 & 7.0 & 37.0 & 29.1 \\
\hline Oesophagus & 65.5 & 68.9 & 3.5 & 3.8 & 22.2 & 7.0 & 153.9 & 17.4 & 35.7 & 38.3 \\
\hline Stomach & 66.1 & 64.3 & 3.6 & 4.7 & 20.3 & 5.9 & | 18.9 & 9.1 & 34.5 & 33.8 \\
\hline Colon & 63.9 & 64.6 & 4.7 & 4.3 & 22.0 & 5.5 & 86.3 & 6.0 & 34.5 & 38.6 \\
\hline Rectum & 66.1 & 66.5 & 4.2 & 6.1 & 20.1 & 3.0 & 94.5 & 14.4 & 37.9 & 29.8 \\
\hline Larynx & 62.3 & 53.7 & 4.1 & 5.7 & 32.9 & 18.3 & 198.2 & 30.0 & 36.5 & 32.7 \\
\hline Lung & 62.1 & 59.4 & 4.2 & 5.2 & 32.3 & 20.5 & 144.9 & 17.8 & 32.7 & 33.5 \\
\hline Breast & - & 59.7 & - & 5.3 & - & 4.1 & - & 12.1 & - & 24.8 \\
\hline Prostate & 70.6 & - & 3.7 & - & 18.0 & - & 96.4 & - & 29.4 & - \\
\hline Bladder & 67.2 & 65.7 & 4.4 & 4.5 & 21.1 & 7.2 & 95.4 & 2.4 & 33.2 & 35.9 \\
\hline Kidney & 61.6 & 58.5 & 4.5 & 5.2 & 20.1 & 6.2 & 113.6 & 3.9 & 25.3 & 33.9 \\
\hline All cases & 64.2 & 61.6 & 4.1 & 5.1 & 26.6 & 5.9 & 141.2 & 11.6 & 33.5 & 29.6 \\
\hline All controls & 64.2 & 60.5 & 4.2 & 4.8 & 18.0 & 4.1 & 98.2 & 8.2 & 24.3 & 19.9 \\
\hline
\end{tabular}


Table 3 Odds ratios of cancer sites for processed meat consumption ${ }^{\mathrm{a}, \mathrm{b}, \mathrm{c}, \mathrm{d}, \mathrm{e}}$

\begin{tabular}{|c|c|c|c|c|}
\hline & & Tertiles & & \\
\hline $\begin{array}{l}\text { Grams per day } \\
\text { Cancer site }\end{array}$ & $\begin{array}{c}\text { I } \leqslant \text { I I.4 } \\
\text { OR } \\
\text { (reference) }\end{array}$ & $\begin{array}{l}\text { II I I.5-28.2 } \\
\text { OR (95\% CI) }\end{array}$ & $\begin{array}{l}\text { III } 28.3+ \\
\text { OR }(95 \% \mathrm{CI})\end{array}$ & $\begin{array}{c}\text { P-value } \\
\text { trend }\end{array}$ \\
\hline Oral/pharynx & & & & \\
\hline $\begin{array}{l}\mathrm{b} \\
\mathrm{c}\end{array}$ & $\begin{array}{c}56 / 844 \\
1.0\end{array}$ & $\begin{array}{c}87 / 844 \\
1.21(0.79-1.87)\end{array}$ & $\begin{array}{c}140 / 844 \\
1.42(0.95-2.13)\end{array}$ & 0.07 \\
\hline $\begin{array}{l}\text { Oesophagus } \\
\text { b } \\
\text { c } \\
\text { d }\end{array}$ & $\begin{array}{c}51 / 844 \\
1.0 \\
1.0\end{array}$ & $\begin{array}{c}76 / 844 \\
1.25(0.81-1.94) \\
2.05(0.90-4.68)\end{array}$ & $\begin{array}{c}107 / 844 \\
1.67(1.08-2.60) \\
3.02(1.40-6.52)\end{array}$ & $\begin{array}{l}0.02 \\
0.004\end{array}$ \\
\hline $\begin{array}{l}\text { Stomach } \\
\text { b } \\
\text { c } \\
\text { d }\end{array}$ & $\begin{array}{c}53 / 844 \\
1.0 \\
1.0\end{array}$ & $\begin{array}{c}95 / 844 \\
1.60(1.02-2.49) \\
3.07(1.58-5.98)\end{array}$ & $\begin{array}{c}126 / 844 \\
1.93(1.25-2.98) \\
4.51(2.34-8.70)\end{array}$ & $\begin{array}{r}0.003 \\
<0.0001\end{array}$ \\
\hline $\begin{array}{c}\text { Colon } \\
b \\
c \\
d\end{array}$ & $\begin{array}{c}34 / 844 \\
1.0 \\
1.0\end{array}$ & $\begin{array}{c}62 / 844 \\
1.76(0.94-3.28) \\
2.25(1.19-4.23)\end{array}$ & $\begin{array}{c}80 / 844 \\
2.01(1.07-3.76) \\
3.53(1.93-6.46)\end{array}$ & $\begin{array}{c}0.03 \\
<0.0001\end{array}$ \\
\hline $\begin{array}{c}\text { Rectum } \\
\text { b } \\
\text { c } \\
\text { d }\end{array}$ & $\begin{array}{c}41 / 844 \\
1.0 \\
1.0\end{array}$ & $\begin{array}{c}65 / 844 \\
1.47(0.85-2.54) \\
2.44(1.17-5.09)\end{array}$ & $\begin{array}{c}79 / 844 \\
1.76(1.03-3.01) \\
3.18(1.54-6.57)\end{array}$ & $\begin{array}{l}0.03 \\
0.001\end{array}$ \\
\hline $\begin{array}{l}\text { Colon/rectum } \\
\text { b } \\
\text { c } \\
\text { d }\end{array}$ & $\begin{array}{c}75 / 844 \\
1.0 \\
1.0\end{array}$ & $\begin{array}{c}127 / 844 \\
1.55(1.01-2.36) \\
2.33(1.42-3.82)\end{array}$ & $\begin{array}{c}159 / 844 \\
1.88(1.23-2.86) \\
3.36(2.08-5.43)\end{array}$ & $\begin{aligned} & 0.004 \\
< & 0.0001\end{aligned}$ \\
\hline $\begin{array}{l}\text { Larynx } \\
\text { b } \\
\text { c }\end{array}$ & $\begin{array}{c}47 / 844 \\
1.0\end{array}$ & $\begin{array}{c}85 / 844 \\
1.75(1.13-2.70)\end{array}$ & $\begin{array}{c}\mid 40 / 844 \\
2.58(|.7|-3.89)\end{array}$ & $<0.0001$ \\
\hline $\begin{array}{l}\text { UADT } \\
\text { e,b } \\
c \\
\text { d }\end{array}$ & $\begin{array}{c}154 / 844 \\
1.0 \\
1.0\end{array}$ & $\begin{array}{c}248 / 844 \\
1.32(0.99-1.76) \\
1.60(0.78-3.31)\end{array}$ & $\begin{array}{c}396 / 844 \\
1.80(1.37-2.38) \\
2.73(1.42-5.26)\end{array}$ & $\begin{array}{c}<0.0001 \\
0.002\end{array}$ \\
\hline $\begin{array}{c}\text { Lung } \\
\text { b } \\
c \\
d\end{array}$ & $\begin{array}{c}176 / 844 \\
1.0 \\
1.0\end{array}$ & $\begin{array}{c}310 / 844 \\
1.43(1.11-1.85) \\
2.26(0.99-5.15)\end{array}$ & $\begin{array}{c}434 / 844 \\
1.82(1.42-2.33) \\
2.54(1.12-5.79)\end{array}$ & $\begin{array}{c}<0.0001 \\
0.02\end{array}$ \\
\hline $\begin{array}{c}\text { Breast } \\
b \\
d\end{array}$ & $\begin{array}{c}127 / 844 \\
1.0\end{array}$ & $\begin{array}{c}161 / 844 \\
1.39(1.04-1.86)\end{array}$ & $\begin{array}{c}173 / 844 \\
1.79(1.83-2.40)\end{array}$ & 0.0001 \\
\hline $\begin{array}{c}\text { Prostate } \\
\text { b } \\
\text { c }\end{array}$ & $\begin{array}{c}75 / 844 \\
1.0\end{array}$ & $\begin{array}{c}134 / 844 \\
1.69(1.22-2.37)\end{array}$ & $\begin{array}{c}136 / 844 \\
1.7 \mid(1.23-2.39)\end{array}$ & 0.002 \\
\hline $\begin{array}{c}\text { Bladder } \\
\text { b } \\
\text { c } \\
\text { d }\end{array}$ & $\begin{array}{c}54 / 844 \\
1.0 \\
1.0\end{array}$ & $\begin{array}{c}98 / 844 \\
1.68(1 .|4-2.5|) \\
2.40(0.86-6.70)\end{array}$ & $\begin{array}{c}103 / 844 \\
1.7 \mid(1.14-2.54) \\
1.97(0.77-5.03)\end{array}$ & $\begin{array}{l}0.01 \\
0.12\end{array}$ \\
\hline $\begin{array}{c}\text { Kidney } \\
\text { b } \\
c \\
\text { d }\end{array}$ & $\begin{array}{c}29 / 844 \\
1.0 \\
1.0\end{array}$ & $\begin{array}{c}38 / 844 \\
0.99(0.53-1.85) \\
2.04(0.85-4.91)\end{array}$ & $\begin{array}{c}47 / 844 \\
1.21(0.65-2.25) \\
2.15(0.90-5.13)\end{array}$ & $\begin{array}{l}0.51 \\
0.07\end{array}$ \\
\hline $\begin{array}{l}\text { Urinary tract } \\
\text { b } \\
\text { c } \\
\text { d }\end{array}$ & $\begin{array}{l}83 / 844 \\
1.0 \\
1.0\end{array}$ & $\begin{array}{c}136 / 844 \\
1.47(1.04-2.07) \\
2.13(1.09-4.15)\end{array}$ & $\begin{array}{c}150 / 844 \\
1.56(1.10-2.21) \\
2.11(1.10-4.04)\end{array}$ & $\begin{array}{l}0.01 \\
0.02\end{array}$ \\
\hline
\end{tabular}

\section{All sites}

\begin{tabular}{lcccc} 
b & $734 / 844$ & $1211 / 844$ & $1574 / 844$ & \\
$c$ & 1.0 & $1.42(1.20-1.69)$ & $1.73(1.46-2.05)$ & $<0.0001$ \\
$d$ & 1.0 & $1.72(1.35-2.19)$ & $2.32(1.82-2.96)$ & $<0.0001$ \\
\hline
\end{tabular}

Abbreviations: $\mathrm{Cl}=$ confidence interval; $\mathrm{OR}=$ odds ratio. ${ }^{\text {a Multivariate adjusted for }}$ age, residence, body mass index, smoking status, smoking cessation, number of cigarettes smoked per day among current smokers, alcohol drinking, maté consumption, total energy, total vegetables and fruits, total white meat, and red meat intakes. 'Number of cases and controls by intake of processed meat (both sexes). ${ }^{C}$ Men. ${ }^{d}$ Women. ${ }^{e}$ Cancer of the upper aerodigestive tract (oral, pharynx, oesophagus, larynx). salami was positively associated with the risk of laryngeal cancer, lung cancer (OR 1.16, 95\% CI 1.07-1.25), breast cancer (OR 1.22, 95\% CI 1.09-1.36), and prostate adenocarcinoma (OR 1.13, 95\% CI 1.01-1.26). Saucisson consumption was directly associated only with gastric cancer (OR 1.25, 95\% CI 1.09-1.44) and hot dog intake was positively associated with most cancer sites, with the exception of breast and prostate cancers. The highest risk for hot dog intake was observed for gastric cancer (OR 1.53, 95\% CI 1.40-1.71). Also, ham consumption was directly associated with cancers of the stomach, colon, rectum, lung, breast, bladder, and kidney, being the highest risks associated with renal cell carcinoma (OR 1.33, 95\% CI 1.13-1.55) and lung cancer (OR 1.28, 95\% CI 1.18-1.40). Finally, air-dried and salted lamb intake was directly associated with cancers of the oesophagus, colon, rectum, lung, breast, prostate, bladder, and kidney. The highest risk for this food item was observed among bladder cancer (OR 1.32, 95\% CI 1.19-1.46).

\section{DISCUSSION}

According to our study, processed meat intake was positively associated with cancers of the oesophagus, stomach, colon, rectum, larynx, lung, breast, prostate, and urinary bladder. Therefore, processed meat could be said to act as a multiorgan carcinogen among humans (World Cancer Research Fund/American Institute for Cancer Research, 2008).

Moreover, our study replicates the findings of previous reports (Larsson et al, 2006; Cross et al, 2007; Santarelli et al, 2008), which strongly suggest that processed meat consumption was associated with an increased risk of gastric and colorectal cancers.

The mechanisms of processed meat are somehow conflictive, but ham, salt (mainly for gastric cancer), aromatic amines, nitrites, and nitrosamines are strong candidates for explaining the effect of cured meats (Santarelli et al, 2008) in the process of carcinogenesis.

Oesophageal cancer, mainly the squamous cell histologic type, has been positively associated with the consumption of air-dried and salted lamb through the presence of nitrosamines. In our report, oesophageal carcinoma displayed an increased risk of $96 \%$, which was highly significant.

Gastric cancer was strongly associated with intake of processed meat, mainly with mortadella, saucisson, hot dog, and ham. All these types of processed meat are high in salt, an enhancing chemical in gastric carcinogenesis (Correa et al, 1985; Chen et al, 1990; Nazario et al, 1993). The comprehensive meta-analysis by Larsson et al (2006) analysed all the studies dealing with stomach cancer and processed meat, both case-control and cohort, and they concluded that proccesed meat is possibly a strong carcinogen in this organ. Several studies also concluded that processed meat is a possible agent in gastric carcinogenesis (De Stefani et al, 2001, 2004; Boeing et al, 1991; Larsson et al, 2006).

According to the prospective study by Cross et al (2007), processed meat increases significantly the risk of developing colorectal cancer. Their findings have been replicated in numerous studies on colorectal cancer and processed meat intake (Goldbohm et al, 1994). According to a study by Goldbohm et al (1994), an intake of $>20 \mathrm{~g}$ per day of processed meat was associated with an increased risk of $72 \%$ for colorectal carcinoma.

Lung cancer has been considered as directly associated with meat intake. In the present study, lung cancer displayed an increased risk of $88 \%$ for consumption of processed meat. When lung cancer risk was examined for the components of the processed meat group, there was significant increase in risk for mortadella, salami, hot dog, ham, and salted meat intakes. Not all studies found an increase in risk of lung cancer. In the EPIC prospective study, Linseisen et al (2011) only found a modest increase of lung cancer for processed meat intake. On the other hand, Lam et al (2009) reported a significant increase 
Table 4 Odds ratios of cancer sites for types of processed meat ${ }^{a, b, c, d}$

\begin{tabular}{|c|c|c|c|c|c|c|c|c|}
\hline Cancer site & OR $(95 \% \mathrm{CI})$ & $\begin{array}{c}\text { Sausage } \\
\text { OR }(95 \% \text { CI) }\end{array}$ & $\begin{array}{r}\text { Mortadella } \\
\text { OR (95\% CI) }\end{array}$ & $\begin{array}{c}\text { Salami } \\
\text { OR }(95 \% \mathrm{CI})\end{array}$ & $\begin{array}{l}\text { Saucisson } \\
\text { OR (95\% CI) }\end{array}$ & $\begin{array}{l}\text { Hot dog } \\
\text { OR }(95 \% \mathrm{CI})\end{array}$ & $\begin{array}{l}\text { Ham } \\
\text { OR }(95 \% \text { CI) }\end{array}$ & $\begin{array}{l}\text { Salted meat } \\
\text { OR (95\% CI) }\end{array}$ \\
\hline \multicolumn{9}{|l|}{ Orallpharynx } \\
\hline b & $0.80(0.66-0.97)$ & $0.91(0.79-1.06)$ & $1.05(0.93-1.18)$ & $1.14(0.99-1.29)$ & $0.92(0.77-1.10)$ & $1.28(1.13-1.43)$ & $1.03(0.89-1.19)$ & $1.07(0.94-1.21)$ \\
\hline \multicolumn{9}{|l|}{ Oesophagus } \\
\hline$b$ & $0.85(0.67-1.07)$ & $0.90(0.75-1.07)$ & $1.09(0.96-1.25)$ & $1.13(0.96-1.31)$ & $0.84(0.67-1.07)$ & $1.40(1.20-1.63)$ & $0.92(0.76-1.11)$ & $1.21(1.07-1.36)$ \\
\hline c & $0.53(0.29-0.98)$ & $1.30(0.9 \mid-1.85)$ & $1.23(0.94-1.62)$ & $0.74(0.50-1.10)$ & $0.97(0.55-1.69)$ & $1.27(0.99-1.64)$ & $1.250 .98-1.59$ & $1.29(0.98-1.69)$ \\
\hline \multicolumn{9}{|l|}{ Stomach } \\
\hline$b$ & $0.64(0.49-0.83)$ & $1.02(0.86-\mid .21)$ & $0.99(0.87-1.14)$ & $0.99(0.86-1.15)$ & $1.22(1.03-1.44)$ & $1.49(1.30-1.70)$ & $0.96(0.81-|| 4)$. & $1.02(0.87-1.19)$ \\
\hline c & $0.72(0.46-1.13)$ & $1.16(0.88-1.53)$ & $1.25(1.01-1.56)$ & $0.76(0.58-0.99)$ & $1.48(1.07-2.04)$ & $1.50(1.23-1.83)$ & $1.24(1.03-1.44)$ & $0.62(0.36-1.07)$ \\
\hline \multicolumn{9}{|l|}{ Colon } \\
\hline$b$ & $0.91(0.65-1.28$ & $1.04(0.81-1.34)$ & $1.17(0.97-1.41)$ & $0.93(0.75-1.15)$ & $0.93(0.69-1.25)$ & $1.17(0.95-1.43)$ & $1.02(0.82-1.26)$ & $1.15(0.95-1.39)$ \\
\hline c & $0.51(0.31-0.82)$ & $1.41(1.08-1.84)$ & $0.99(0.80-1.23)$ & $0.93(0.72-1.20)$ & $0.98(0.64-1.50)$ & $1.36(1.12-1.65)$ & $1.30(1.09-1.56)$ & $1.41(1.17-1.70)$ \\
\hline \multicolumn{9}{|l|}{ Rectum } \\
\hline$b$ & $0.67(0.49-0.94)$ & $0.91(0.73-1.13)$ & $1.05(0.89-1.24)$ & $1.19(1.00-1.41)$ & $0.85(0.65-1.10)$ & $1.34(1.13-1.59)$ & $0.99(0.83-1.20)$ & $1.23(1.06-1.42)$ \\
\hline c & $0.82(0.48-1.38)$ & $1.05(0.75-1.47)$ & $0.90(0.68-1.20)$ & $0.93(0.68-1.26)$ & $0.91(0.55-1.49)$ & $1.31(1.04-1.65)$ & $1.35(1.09-1.68)$ & $1.25(0.96-1.63)$ \\
\hline \multicolumn{9}{|l|}{ Colon/rectum } \\
\hline b & $0.77(0.60-0.99)$ & $0.98(0.82-1.16)$ & $1.10(0.97-1.25)$ & $1.08(0.94-1.24)$ & $0.88(0.72-1.08)$ & $1.28(1.11-1.46)$ & $1.01(0.87-1.17)$ & $1.19(1.05-1.35)$ \\
\hline c & $0.61(0.42-0.88)$ & $1.25(1.01-\mid .56)$ & $0.96(0.80-1.15)$ & $0.93(0.76-1.14)$ & $0.94(0.67-1.33)$ & $1.33(1.15-1.57)$ & $1.33(1.14-1.54)$ & $1.36(1.15-1.61)$ \\
\hline \multicolumn{9}{|l|}{ Larynx } \\
\hline $\mathrm{b}$ & $0.77(0.62-0.96)$ & $1.06(0.90-1.35)$ & $1.16(1.03-1.30)$ & $1.13(0.99-1.30)$ & $1.03(0.87-\mid .22)$ & $1.26(1.10-1.44)$ & $0.92(0.78-1.07)$ & $1.03(0.90-1.17)$ \\
\hline \multicolumn{9}{|l|}{ UADT } \\
\hline$d, b$ & $0.79(0.68-0.92)$ & $0.94(0.84-1.05)$ & $1.08(0.99-1.17)$ & $1.11(1.01-1.21)$ & $0.95(0.84-1.08)$ & $1.30(1.18-1.43)$ & $0.96(0.86-1.06)$ & $1.08(0.99-1.19)$ \\
\hline c & $0.53(0.3 \mid-0.92)$ & $1.42(1.04-1.92)$ & $1.28(0.98-1.57)$ & $0.76(0.55-1.05)$ & $0.81(0.47-1.4 I)$ & $1.28(|.03-| .5 \mid)$ & I.I0 (0.89-1.36) & $1.19(0.9 \mid-1.54)$ \\
\hline \multicolumn{9}{|l|}{ Lung } \\
\hline$b$ & $0.87(0.75-0.99)$ & $0.86(0.77-0.95)$ & $1.05(0.97-1.14)$ & I.II (1.01-1.22) & 0.91 (0.80-1.03) & $1.26(1.15-1.38)$ & $1.28(1.16-1.4 \mid)$ & $1.29(1.19-1.40)$ \\
\hline c & $0.72(0.40-1.68)$ & $1.28(0.90-1.82)$ & $1.36(1.04-1.77)$ & $0.94(0.69-1.29)$ & $0.75(0.49-1.25)$ & $0.96(0.74-1.25)$ & $1.21(0.95-1.55)$ & $1.18(0.89-1.56)$ \\
\hline \multicolumn{9}{|l|}{ Breast } \\
\hline c & $0.94(0.75-1.18)$ & $0.97(0.84-1.12)$ & $1.27(1.1$ I-1.47) & $1.12(0.99-1.37)$ & $1.05(0.87-1.28)$ & $0.88(0.79-0.98)$ & $1.12(1.01-1.25)$ & $1.22(1.06-1.40)$ \\
\hline \multicolumn{9}{|l|}{ Prostate } \\
\hline$b$ & $0.84(0.68-1.04)$ & $1.01(0.87-1.17)$ & $1.27(1.14-1.43)$ & $1.09(0.96-1.23)$ & $0.80(0.67-0.96)$ & $0.79(0.68-0.92)$ & $0.98(0.85-1.12)$ & $1.25(1.13-1.37)$ \\
\hline \multicolumn{9}{|l|}{ Bladder } \\
\hline$b$ & $0.62(0.48-0.82)$ & $1.01(0.85-1.19)$ & $0.91(0.80-1.04)$ & $1.08(0.93-1.25)$ & $0.64(0.49-0.84)$ & $1.33(1.16-1.53)$ & $1.23(1.07-1.41)$ & $1.83(1.18-1.49)$ \\
\hline$c$ & $0.59(0.29-1.21)$ & $1.70(1.08 .2 .66)$ & $0.95(0.64-1.49)$ & $0.56(0.29-1.09)$ & $1.06(0.49-2.21)$ & $1.07(0.74-1.56)$ & I.II (0.78-1.59) & $1.69(1.26-2.29)$ \\
\hline \multicolumn{9}{|l|}{ Kidney } \\
\hline$b$ & $0.54(0.33-0.89)$ & $0.85(0.65-1.11)$ & $1.08(0.88-1.33)$ & $1.02(0.82-1.28)$ & $0.48(0.27-0.86)$ & $0.90(0.70-1.14)$ & $1.35(1.10-1.65)$ & $1.22(0.99-1.51)$ \\
\hline C & $0.51(0.24-1.10)$ & $1.44(0.96-2.15)$ & $0.65(0.43-0.98)$ & $1.13(0.78-1.62)$ & $1.55(0.90-2.68)$ & $1.22(0.90-\mid .64)$ & $1.28(0.97-1.70)$ & $1.66(1.24-2.23)$ \\
\hline \multicolumn{9}{|l|}{ Urinary tract } \\
\hline$b$ & $0.62(0.49-0.79)$ & $0.94(0.81-1.10)$ & $0.97(0.86-1.08)$ & $1.05(0.92-1.19)$ & $0.61(0.48-0.79)$ & $1.21(1.07-1.37)$ & $1.27(1.13-1.43)$ & $1.30(1.17-1.44)$ \\
\hline c & $0.58(0.34-0.97)$ & $1.52(1.12-2.05)$ & $0.80(0.60-1.07)$ & $0.90(0.65-1.24)$ & $1.17(0.74-1.85)$ & $1.17(0.92-1.49)$ & $1.18(0.94-1.48)$ & $1.60(1.37-2.09)$ \\
\hline \multicolumn{9}{|l|}{ All sites } \\
\hline$b$ & $0.79(0.7 \mid-0.87)$ & $0.93(0.86-1.00)$ & $1.09(1.03-1.15)$ & $1.12(1.05-1.19)$ & $0.92(0.85-1.01)$ & $1.21(1.13-1.29)$ & $1.08(1.01-1.15)$ & $1.21(1.14-1.29)$ \\
\hline C & $0.78(0.65-0.93)$ & $1.09(0.97-1.22)$ & $1.20(1.09-1.32)$ & $1.0 \mid(0.9|-| . \mid 2)$ & $1.02(0.87-1.20)$ & $1.08(0.99-1.17)$ & $1.18(1.09-1.28)$ & $1.70(1.16-1.46)$ \\
\hline
\end{tabular}

Abbreviations: $\mathrm{Cl}=$ confidence interval; $\mathrm{OR}=$ odds ratio. ${ }^{\mathrm{a}}$ Multivariate adjusted for age, residence, body mass index, smoking status, smoking cessation, number of cigarettes smoked per day among current smokers, alcohol drinking, maté consumption, total energy, total vegetables and fruits, total white meat, red meat intakes, bacon, sausage, mortadella, salami, saucisson, hot dog, ham, and salted meat. bMen. 'Women. ${ }^{\mathrm{d} C a n c e r}$ of the upper aerodigestive tract (oral, pharynx, oesophagus, larynx).

in the risk of lung cancer of $71 \%$ for weekly consumption of processed meat.

Female breast cancer is the higher malignancy among Uruguayan women with an ASIR of 116 persons per 100000 (Parkin et al, 2002). In fact, this rate is the highest in the world (Parkin et al, 2002). Studies conducted in Uruguay reported elevated risks for red meat intake (Ronco et al, 1996; De Stefani et al, 1997; Ronco et al, 2006). In the present study, breast cancer showed an increased risk of $82 \%$ for high intake of processed meat. In a study by Pala et al (2009), postmenopausal women afflicted with breast cancer showed an increased risk of $13 \%(P=0.06)$, whereas the study by Aune et al (2009) displayed an increased risk of 53\% for consumption of processed meat among women afflicted with breast cancer.

Prostate cancer is the second malignancy in frequency among Uruguay men, following lung cancer (Parkin et al, 2002). 
According to the report by the World Cancer Research Fund (World Cancer Research Fund/American Institute for Cancer Research, 2008), processed meat intake has a suggestive role among those men afflicted with this malignancy. In the present study, patients with prostate cancer showed an increased risk of $72 \%$ per $28 \mathrm{~g}$ per day. Sinha et al (2009) studied the risk of advanced prostate cancer and found an increased risk of $32 \%$ per $25 \mathrm{~g}$ per $1000 \mathrm{cal}$. Thus, our findings replicate those reported by Sinha et al (2009).

In our study, bladder cancer showed a significant increased risk for both sexes with a magnitude similar to that observed for prostate cancer. In a prospective study by Michaud et al (2006), frequent consumption of bacon was positively associated with a relative risk of 2.1 ( $P$-value for trend $=0.006)$. Furthermore, this association was stronger among never smokers. On the contrary, in our study bacon intake was inversely associated with the risk of bladder cancer. This difference could be explained by the low consumption of bacon among the Uruguayan population (mean intake of bacon: $1.1 \mathrm{~g}$ daily). On the contrary, we found rather elevated risks for bladder cancer in our study, directly associated with the intake of hot dogs, ham, and salted meat.

Like other case-control studies, the present study is subject to several potential biases, such as selection bias and recall bias. Nevertheless, the Uruguayan population is mainly unaware of the

\section{REFERENCES}

Aune D, De Stefani E, Ronco AL, Boffetta P, Deneo-Pellegrini H, Acosta G, Mendilaharsu M (2009) Meat consumption and cancer risk: a casecontrol study in Uruguay. Asian Pac J Cancer Prev 10: 429-436

Boeing H, Frentzel-Beyme R, Berger M, Berndt V, Göres W, Körner M, Lohmeier R, Menarcher A, Männl HFK, Meinhardt M, Müller R, Ostermeier H, Paul F, Schwemmle K, Wagner KH, Wahrendorf J (1991) Case-control study on stomach cancer in Germany. Int J Cancer 47(6): 858-864

Chen VW, Abu-Elyazeed RR, Zavala D, Ktsanes VK, Haenszel W, Cuello C, Montes G, Correa P (1990) Risk factors of gastric precancerous lesions in a high-risk Colombian population. Nutr Cancer 13: 59-65

Correa P, Fontham E, Pickle LW, Chen V, Lin Y, Haenszel W (1985) Dietary determinants of gastric cancer in south Louisiana inhabitants. $J$ Natl Cancer Inst 75: 645-654

Cross AJ, Leitzmann MF, Gail MH, Hollenbeck AR, Schatzkin A, Sinha R (2007) A prospective study of red and processed meat intake in relation to cancer risk. PloS Med 4: e325

De Stefani E, Ronco A, Brennan P, Boffetta P (2001) Meat consumption and risk of stomach cancer in Uruguay. Nutr Cancer 40: 103-107

De Stefani E, Correa P, Boffetta P, Deneo-Pellegrini H, Ronco AL, Mendilaharsu M (2004) Dietary patterns and risk of gastric cancer: a case-control study in Uruguay. Gastric Cancer 7: 211-220

De Stefani E, Ronco AL, Mendilaharsu M (1997) Meat intake, heterocyclic amines, and risk of breast cancer: a case-control study in Uruguay. Cancer Epidemiol Biomarkers Prev 6: 573-581

Goldbohm RA, van den Brandt PA, van't Veer P, Brants HAM, Dorant E, Sturmans F, Hermus RJJ (1994) A prospective cohort study on the relation between meat consumption and the risk of colon cancer. Cancer Res 54: 718-723

Lam TK, Cross AJ, Consonni D, Randi G, Bagnardi V, Bertazzi PA, Caporaso NE, Sinha R, Subar AF, Landi MT (2009) Intakes of red meat, processed meat, and meat-mutagens increase lung cancer risk. Cancer Res 69: 932-939

Larsson SC, Orsini N, Wolk A (2006) Processed meat consumption and stomach cancer risk: a meta-analysis. J Natl Cancer Inst 98: 1078-1087

Linseisen J, Rohrmann S, Bueno-de-Mesquita B, Büchner FL, Boshuizen HC (2011) Consumption of meat and fish and risk of lung cancer: results from the EPIC. Cancer Causes Control 22: 909-919

Matos E, Brandani A (2002) Review on meat consumption and cancer in South America. Mutat Res 506-507: 243-249 potential danger of a high consumption of processed meat. This also applies to interviewers. Our study has several strengths. In the first place, the statistical power of the study is a strength. Second, all the cases were microscopically validated by expert pathologists. Finally, the high response rate for cases and controls is another significant strength of the study.

In summary, we conducted a sizeable case-control study on the role of processed meat consumption in several cancer sites. The results were consistently associated with an elevation in risk for most cancer sites, suggesting that processed meat consumption could be a possible risk factor for various cancer sites.

\section{ACKNOWLEDGEMENTS}

The present study was funded by the International Agency for Research on Cancer, Lyon, France. This work was supported by a grant from International Agency for Research on Cancer, Lyon, France.

\section{Conflict of interest}

The authors declare no conflict of interest.

Michaud DS, Holick CN, Giovannucci E, Stampfer MJ (2006) Meat intake and bladder cancer risk in 2 prospective cohort studies. Am J Clin Nutr 84: 1177-1183

Nazario CM, Szklo M, Diamond E, Román-Franco A, Climent C, Suarez E, Conde JG (1993) Salt and gastric cancer: a case-control study in Puerto Rico. Int J Epidemiol 22: 790-797

Pala V, Krogh V, Berrino F, Sieri S, Grioni S, Tjønneland A, Olsen A, Jakobsen MU, Overvad K, Clavel-Chapelon F, Boutron-Ruault MC, Romieu I, Linseisen J, Rohrmann S, Boeing H, Steffen A, Trichopoulou A, Benetou V, Naska A, Vineis P, Tumino R, Panico S, Masala G, Agnoli C, Engeset D, Skeie G, Lund E, Ardanaz E, Navarro C, Sánchez MJ Amiano P, Svatetz CA, Rodriguez L, Wirfält E, Manjer J, Lenner P, Hallmans G, Peeters PH, van Gils CH, Bueno-de-Mesquita HB, van Duijnhoven FJ, Key TJ, Spencer E, Bingham S, Khaw KT, Ferrari P, Byrnes G, Rinaldi S, Norat T, Michaud DS, Riboli E (2009) Meat, eggs, dairy products, and risk of breast cancer in the EPIC cohort. Am J Clin Nutr 90: 602-612

Parkin DM, Whelan SL, Ferlay J, Teppo L, Thomas DB. (editors) (2002) Cancer Incidence in Five Continents. Vol. VIII, IARC Scientific Publications No. 155: Lyon, France

Ronco AL, De Stefani E, Mendilaharsu M (1996) Meat, fat and the risk of breast cancer: a case-control study from Uruguay. Int J Cancer 65: 328-331

Ronco AL, De Stefani E, Boffetta P (2006) Food patterns and risk of breast cancer: a factor analysis study in Uruguay. Int J Cancer 119: 1672-1678

Rothman KJ, Greenland S, Lash TL (2008) Modern Epidemiology. 3rd edn. Lippincott Williams \& Wilkins: Philadelphia

Santarelli RL, Pierre F, Corbet DE (2008) Processed meat and colorecta cancer. A review of epidemiologic and experimental evidence. Nutr Cancer 60: 131-144

Sinha R, Park Y, Graubard BI, Leitzmann MF, Hollenbeck A, Schatzkin A, Cross AJ (2009) Meat and meat-related compounds and risk of prostate cancer in a large prospective cohort study in the United States. Am J Epidemiol 170: 1165-1177

StataCorp (2008) Stata Statistical Software. Release 11. College Station: TX USA

World Cancer Research Fund/American Institute for Cancer Research (2008) Food, Nutrition, Physical Activity, and the Prevention of Cancer: a Global Perspective. AICR: Washington DC

Willett WC (1998) Nutritional Epidemiology. 2nd edn. Oxford University Press: Oxford, pp 332-338

This work is published under the standard license to publish agreement. After 12 months the work will become freely available and the license terms will switch to a Creative Commons Attribution-NonCommercial-Share Alike 3.0 Unported License. 